\title{
BRCAA1 monoclonal antibody conjugated fluorescent magnetic nanoparticles for in vivo targeted magnetofluorescent imaging of gastric cancer
}

Kan Wang ${ }^{1 \dagger}$, Jing Ruan ${ }^{1 \dagger}$, Qirong Qian ${ }^{2 *}$, Hua Song ${ }^{1}$, Chenchen Bao ${ }^{1}$, Xueqing Zhang ${ }^{1}$, Yifei Kong ${ }^{1}$, Chunlei Zhang ${ }^{1}$, Guohan $\mathrm{Hu}^{2}$, Jian $\mathrm{Ni}^{1}$ and Daxiang Cui ${ }^{* *}$

\begin{abstract}
Background: Gastric cancer is 2th most common cancer in China, and is still the second most common cause of cancer-related death in the world. How to recognize early gastric cancer cells is still a great challenge for early diagnosis and therapy of patients with gastric cancer. This study is aimed to develop one kind of multifunctional nanoprobes for in vivo targeted magnetofluorescent imaging of gastric cancer.

Methods: BRCAA1 monoclonal antibody was prepared, was used as first antibody to stain 50 pairs of specimens of gastric cancer and control normal gastric mucous tissues, and conjugated with fluorescent magnetic nanoparticles with $50 \mathrm{~nm}$ in diameter, the resultant BRCAA1-conjugated fluorescent magnetic nanoprobes were characterized by transmission electron microscopy and photoluminescence spectrometry, as-prepared nanoprobes were incubated with gastric cancer MGC803 cells, and were injected into mice model loaded with gastric cancer of $5 \mathrm{~mm}$ in diameter via tail vein, and then were imaged by fluorescence optical imaging and magnetic resonance imaging, their biodistribution was investigated. The tissue slices were observed by fluorescent microscopy, and the important organs such as heart, lung, kidney, brain and liver were analyzed by hematoxylin and eosin (HE) stain method.

Results: BRCAA1 monoclonal antibody was successfully prepared, BRCAA1 protein exhibited over-expression in $64 \%$ gastric cancer tissues, no expression in control normal gastric mucous tissues, there exists statistical difference between two groups $(P<0.01)$. The BRCAA1-conjugated fluorescent magnetic nanoprobes exhibit very lowtoxicity, lower magnetic intensity and lower fluorescent intensity with peak-blue-shift than pure FMNPs, could be endocytosed by gastric cancer MGC803 cells, could target in vivo gastric cancer tissues loaded by mice, and could be used to image gastric cancer tissues by fluorescent imaging and magnetic resonance imaging, and mainly distributed in local gastric cancer tissues within $12 \mathrm{~h}$ post-injection. HE stain analysis showed that no obvious damages were observed in important organs.
\end{abstract}

Conclusions: The high-performance BRCAA1 monoclonal antibody-conjugated fluorescent magnetic nanoparticles can target in vivo gastric cancer cells, can be used for simultaneous magnetofluorescent imaging, and may have

\footnotetext{
* Correspondence: qianqr@163.com; daxiangcui@yahoo.com

+ Contributed equally

'Department of Bio-nano Science and Engineering, National Key Laboratory

of Nano/Micro Fabrication Technology, Key Laboratory for Thin Film and

Microfabrication of Ministry of Education, Institute of Micro-Nano Science

and Technology, Shanghai Jiao Tong University, 800 Dongchuan Road,

Shanghai 200240, China

${ }^{2}$ Department of Surgery, Changzheng Hospital affiliated to Second Military

Medical University, 151 Fengyang Road, Shanghai 20003, China

Full list of author information is available at the end of the article
} 


\section{Background}

Gastric cancer was once the second most common cancer in the word [1]. Up to date, in the United States, stomach malignancy is currently the 14th most common cancer, and 2 th most common cancer in China [2,3]. Gastric cancer is still the second most common cause of cancer-related death in the world, and remains difficult to cure because most patients present with advanced disease. Therefore, how to recognize, track or kill early gastric cancer cells is very key for early diagnosis and therapy of patients with gastric cancer.

Up to date, looking for biomarkers closely associated with gastric cancer is still an important task. Since 1998, we have been being tried to establish an early gastric cancer pre-warning system [4], and hope to use this pre-warning system to detect early gastric cancer cells to recognize the patients with early gastric cancer. Although some differently-expressed genes associated with early gastric cancer were identified $[5,6]$, no one gene can be confirmed to be specific biomarker of gastric cancer. Therefore, in order to recognize early gastric cancer cells, we only select potential biomarkers associated with gastric cancer, and combine nanoparticles and molecular imaging techniques, try to find in vivo early gastric cancer cells by in vivo tumor targeted imaging. In our previous work, we screened out and cloned BRCAA1 gene (breast cancer associated antigen 1 gene) from breast cancer cell line MCF-7cells [AF208045, also called ARID4B (AT-rich interactive domain-containing protein $4 \mathrm{~B})]$, and identified its antigen epitope peptide SSKKQKRSHK $[7,8]$. We also prepared BRCAA1 polyclonal antibody, and observed that the BRCAA1 protein exhibited over-expression in almost $65 \%$ clinical specimens of gastric cancer tissues [9-11]. We also observed that BRCAA1 antigen is over-expressed in gastric cancer cell lines such as MKN-1, MKN-74, SGC-7901, KATO-III and MGC803 cells. Therefore, we predict that BRCAA1 protein may be one potential targeting molecule for in vivo gastric cancer cells.

In recent years, molecular imaging technologies based on multi-functional nanoprobes have made great progress. For example, nanoparticles such as quantum dots, magnetic nanoparticles and gold nanorods, etc. have been used for molecular imaging [12-19]. So far several small animal imaging technologies have been developed such as optical imaging (OI) of bioluminescence (BLI), fluorescence (FLI) and of intravital microscopy (IVM), micro-PET, MRI and CT [20-26]. Among all these technologies, how to improve their spatial resolution and tissue depth sensitivity is a great challenge. So far in vivo tumor tissues with over $1 \mathrm{~cm}$ in diameter can be easily identified by CT, MRI, PET and bioluminescence imaging, tumors with less than or equal to $5 \mathrm{~mm}$ in diameter is very difficult to be found in clinical patients. In our previous reports, photosensitizer-conjugated magnetic nanoparticles were successfully used for in vivo simultaneous magnetofluorescent imaging and targeting therapy [27]. However, the targeting ability of nanoprobes was highly dependent on magnetic nanoparticles. We also prepared a multifunctional Ribonuclease-Aconjugated CdTe quantum dot cluster nanosystem for synchronous cancer imaging and therapy [28], the targeting ability of as-prepared nanoprobes is dependent on RGD peptide. Some studies show that HER-2 protein exhibits abnormal expression in 6-35\% gastric cancer tissues $[29,30]$, and has been used as the therapeutic target for clinical patients with gastric cancer [31], therefore, HER-2 protein owns great potential in imaging and therapy of gastric cancer. However, up to date, no report shows that targeted imaging and therapy of in vivo gastric cancer is based on biomarkers associated with gastric cancer.

In recent years, we controllably prepared silica-coated quantum dots and super-paramagnetic nanoparticle composites(FMNPs) with strong fluorescent signals and excellent magnetic properties, and have used them for bio-labeling, tracking stem cells, bio-separation, targeting imaging and hyperthermia of tumors [29-32], we also observed that as-prepared nanoparticles own good biocompatibility and stability [33-38].

In this paper, we fully use the advantages of FMNPs and BRCAA1 antigen, prepared monoclonal antibody against BRCAA1 protein, and prepared BRCAA1 monoclonal antibody-conjugated fluorescent magnetic nanoprobes (BRCAA1-FMNPs), employed nude mice model loaded with gastric cancer of $5 \mathrm{~mm}$ in diameter and IVIS imaging system and Magnetic Resonance Imaging, investigated the feasibility of as-prepared nanoprobes for non-invasive in vivo targeted dual modal imaging of gastric cancer. Results show that as-prepared nanoprobes can be used for in vivo dual-model imaging of gastric cancer, and may have great potential in applications such as dual-model imaging and local thermal therapy of early gastric cancer in near future. 
Table 1 Titers of BRCAA1 Monoclonal Antibodies in Ascites Fluid Induced by Hybridoma Clone Cells by ELISA

\begin{tabular}{ccccc}
\hline & \multicolumn{5}{c}{ Antibody titer* } \\
\cline { 2 - 5 } Clone & BRCAA1 (C)-OVA ** & BRCAA1 (C)-BSA ** & BSA ** & OVA ** \\
\hline S-200-5 & $1,024,000$ & $1,024,000$ & $<1,000$ & $<1,000$ \\
S-335-5 & 128,000 & 512,000 & $<1,000$ & $<1,000$ \\
\hline
\end{tabular}

*The reciprocal of ascites fluid dilution, the first dilution of ascites fluid was 1:1,000.

**The antigens were coated on ELISA plate.

\section{Results and Discussion}

\section{Characterization of anti-BRCAA1 monoclonal Antibody}

As shown in Table 1, we successfully obtained two positive clone cell lines S-200-5 and S-335-5, their titers were different, finally we selected the anti-BRCAA1 monoclonal antibody from S-200-5 cell line as the first antibody to stain gastric cancer tissues and control tissues. We found that BRCAA1 protein exhibited overexpression in $64 \%$ gastric cancer tissues, no expression in normal control gastric mucous tissues, as shown in Figure 1, there exists statistical difference between two group $(P<0.01)$. This result is almost identical to our previous report [4,9-11], which highly suggest that BRCAA1 antigen may be selected as the potential target for most gastric cancer, if as-prepared nanoprobes may recognize $64 \%$ patients with early gastric cancer, it will be very useful for diagnosis and therapy of clinical gastric cancer patients.

Preparation and Characterization of BRCAA1- FMNPs nanoprobes

As shown in Figure 2A, prepared FMNPs were composed of silica-wrapped CdTe and magnetic nanoparticles, their size were $50 \mathrm{~nm}$ or so in diameter. As shown in Figure 2D, after FMNPs were conjugated with anti-BRCAA1 antibody, as-prepared nanoprobes' photoluminescence (PL) intensity was lower than that of FMNPs, exhibiting left-shift of $40 \mathrm{~nm}$, which was due to decrease of the polarization rate of the surrounding molecules, and resulting in the decrease of stokes displacement, finally resulting in a blue shift in the emission spectra. Similarly, magnetic intensity of as-prepared nanoprobes was also lower than FMNPs.

In the course of preparing BRCAA1-FMNPs nanoprobes, we found that surface functionalization of FMNPs was very key to conjugate anti-BRCAA1 antibody with FMNPs via covalent bond. As shown in Figure 2C, different functional groups of FMNPs have different zetapotential values. FMNPs had negative $\mathrm{Si}$-O-group, their zeta-potential value was $-34.05 \mathrm{mV}$, the FMNPs with amino group had positive zeta-potential value of 24.80 $\mathrm{mV}$, FMNPs with carboxyl group had negative zetapotential value of $-30.50 \mathrm{mV}$. We observed that carboxyl groups on the surface of FMNPs conjugated with antiBRCAA1 antibody easier than amino groups on the surface of FMNPs. As shown in Table 2, the average coupling rate of anti-BRCAA1 antibody with FMNPs$\mathrm{COOH}$ was $80.28 \%$.

\section{As-prepared nanoprobes for in vitro targeted gastric} cancer cells

Targeting ability of as-prepared nanoprobes in vitro were observed by fluorescence microscope and calculated by FACSCalibur Flow cytometer. As shown in Figure 3, FMNPs randomly dispersed in the inner of the cytoplasm, and anti-BRCAA1-FMNPs nanoprobes existed around the

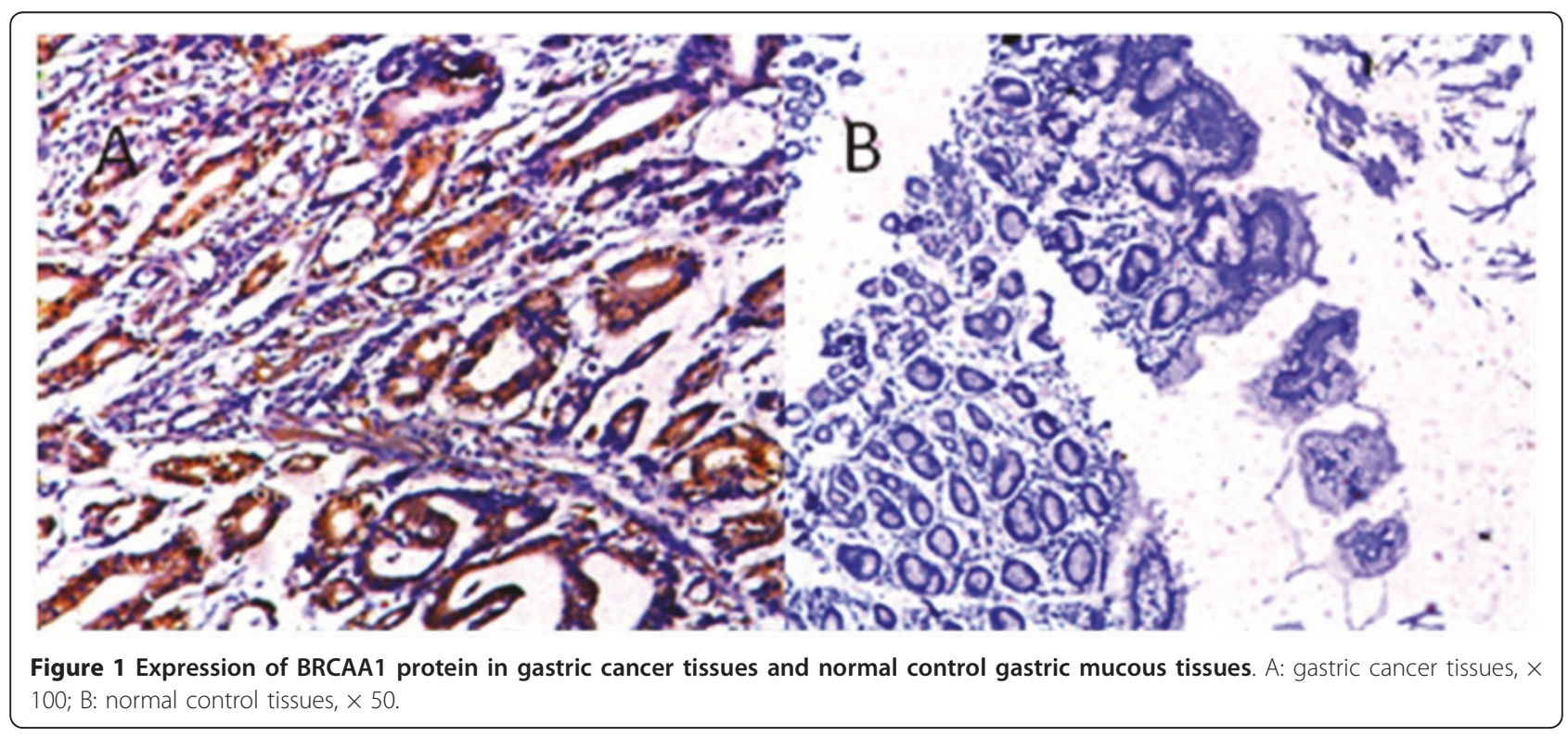




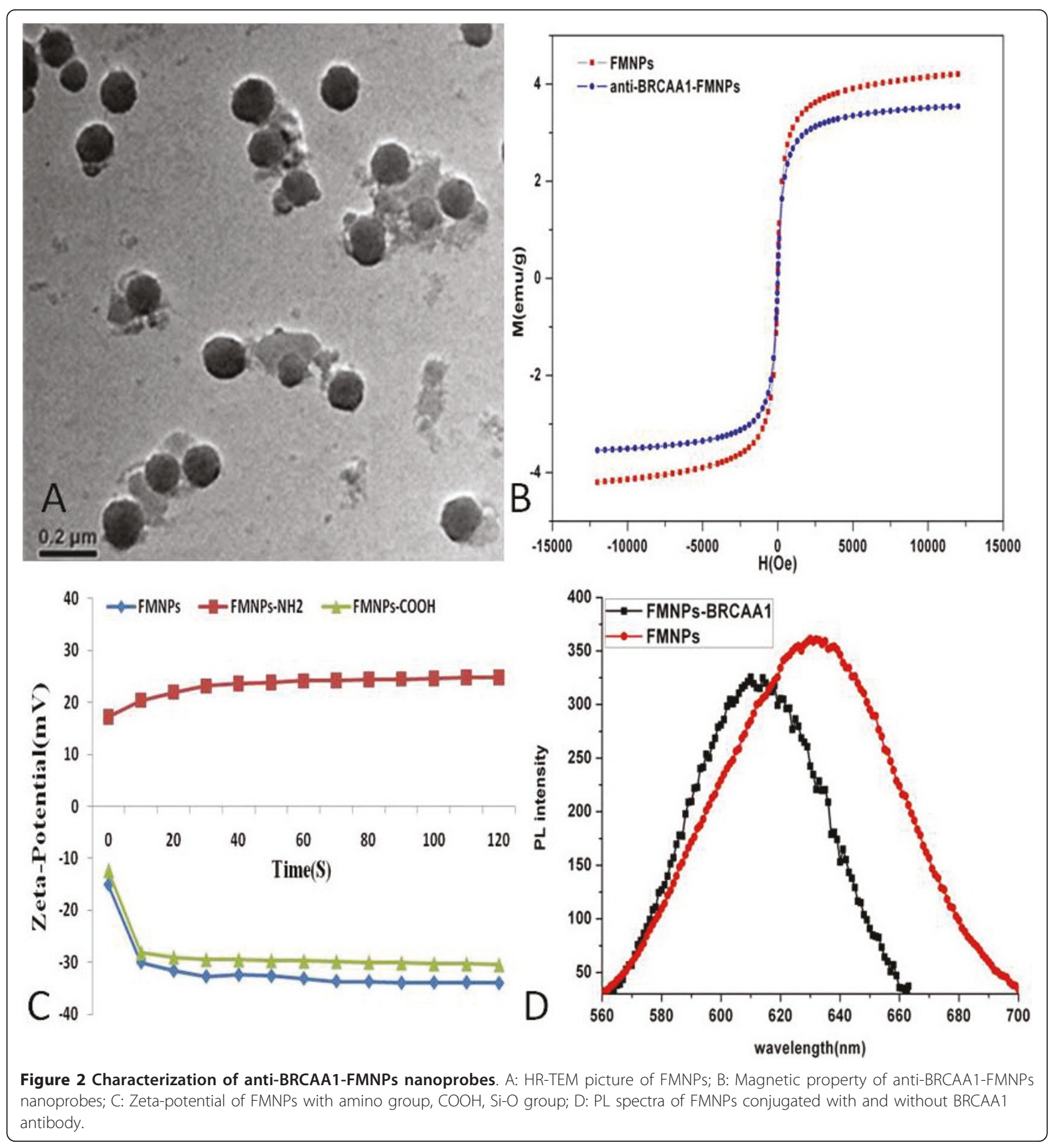

Table 2 Coupling rate measurement of FMNPs-anti-BRCAA1 antibody

\begin{tabular}{llll}
\hline $\begin{array}{l}\text { Total concentration of the anti-BRCAA1 } \\
\text { antibody }(\mathbf{n g} / \boldsymbol{\mu L})\end{array}$ & $\begin{array}{l}\text { The concentration of anti-BRCAA1 antibody in residual reaction } \\
\text { mixture }(\mathbf{n g} / \boldsymbol{\mu L})\end{array}$ & $\begin{array}{l}\text { Coupling rate } \\
(\%)\end{array}$ \\
\hline $1 \quad 1000.0$ & 197.3 & 80.27 \\
\hline 21000.0 & 191.2 & 80.88 \\
\hline $3 \quad 1000.0$ & 203.0 & 79.70 \\
\hline
\end{tabular}




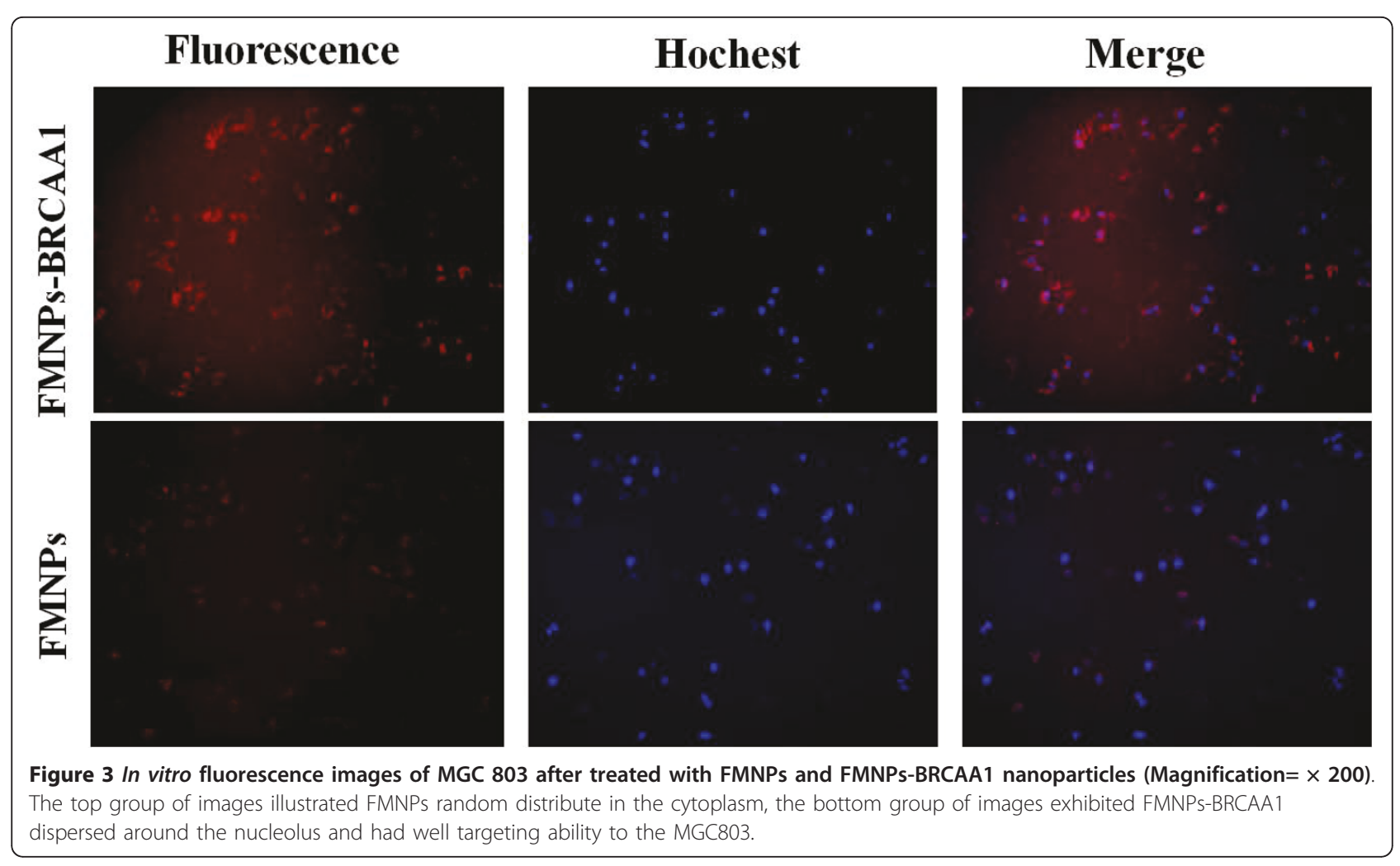

nucleolus. Both FMNPs and prepared BRCAA1-FMNPs nanoprobes can enter into the cytoplasm of MGC803 cells after $4 \mathrm{~h}$ incubation with MGC803 cells, as shown in Figure 4A, FMNPs could label 25.23\% MGC803 cells, the remain $74.77 \%$ cells could not be labeled. As shown in Figure 4B, 45.92\% MGC803 cells could be labeled by the BRCAA1-FMNPs nanoprobes. When FMNPs and antiBRCAA1-FMNPs nanoprobes were respectively incubated

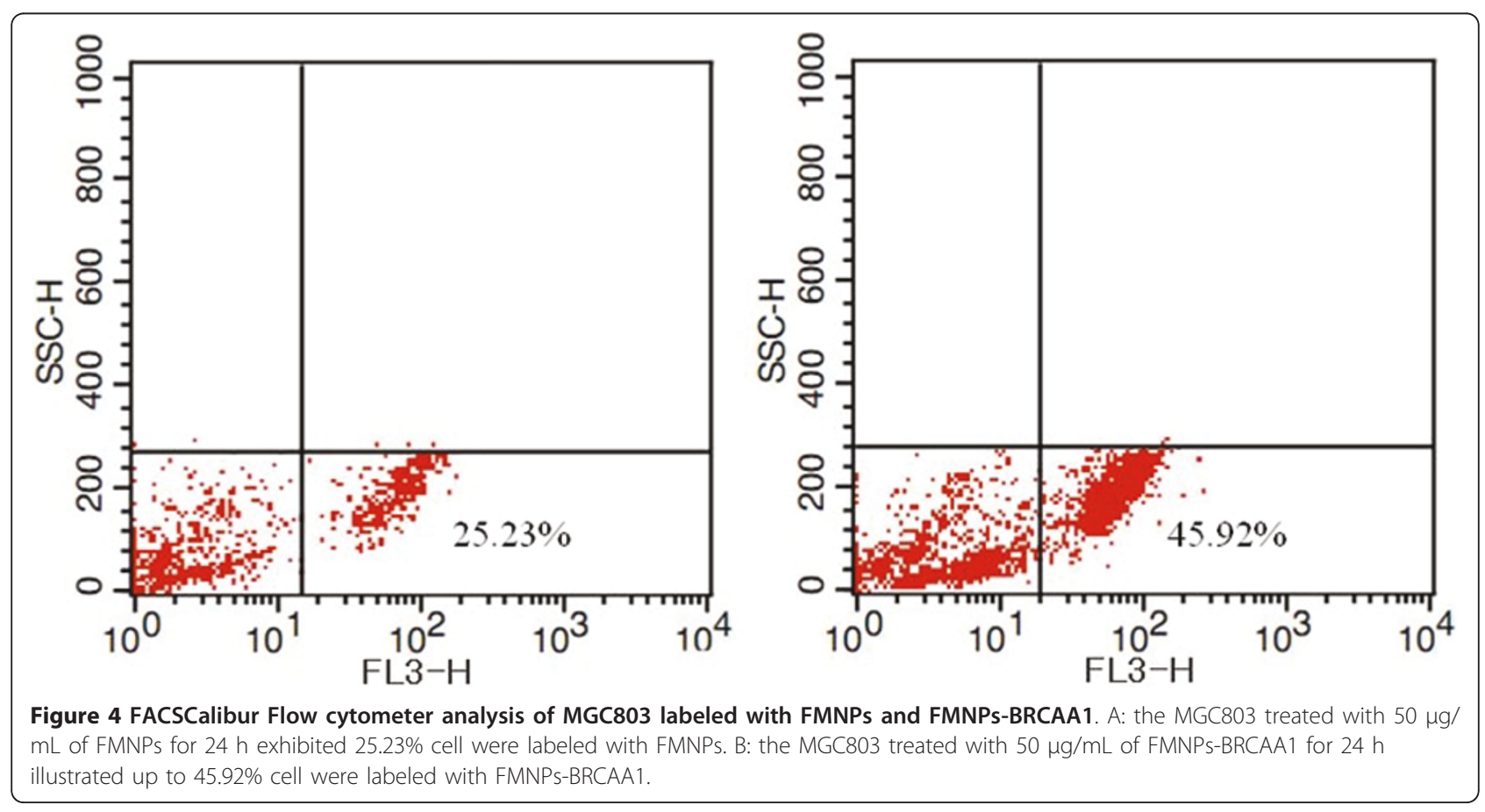


with MGC803 cells and human fibroblast cells for $0.5 \mathrm{~h}$, we observed a lot of anti-BRCAA1-FMNPs nanoprobes entered into MGC803 cells, few nanoprobes entered into human fibroblast cells, few FMNPs could enter into MGC803 cells and human fibroblast cells, which highly suggest that anti-BRCAA1-FMNPs nanoprobes can target MGC803 cells specifically. The Magnetic Resonance imaging of MGC803 cells and human fibroblast cells incubated with anti-BRCAA1-FMNPs for $4 \mathrm{~h}$ were shown in Figure 5, MGC803 cells exhibited strong magnetic signal than human fibroblast cells (HDF), which also showed that the prepared nanoprobes can target MGC803 cells specifically.

\section{As-prepared nanoprobes for fluorescent imaging of in vivo gastric cancer cells}

To evaluate tumor targeted properties of anti-BRCAA1FMNPs nanoprobes, nude mice models loaded with MGC-803 gastric cancer cells were prepared and monitored under a non-invasive manner for $12 \mathrm{~h}$ by using IVIS fluorescence imaging system.

By monitoring real-time fluorescence intensity in the whole body, the tumor-targeting character of the antiBRCAA1-FMNPs probe was easily determined in the nude mice loaded with gastric cancer MGC803 cells. As shown in Figure 6A, the whole animals produced fluorescent signals within 30 min of post-injection of nanoprobes, the subcutaneous tumor tissues could be clearly delineated from the surrounding background tissue between $1 \mathrm{~h}$ and $12 \mathrm{~h}$ post-injection, with maximum contrast occurring at $6 \mathrm{~h}$ post-injection. Strong fluorescence signal was still be detected in the tumor site at 6 $\mathrm{h}$ post-injection, which indicated that the anti-BRCAA1-

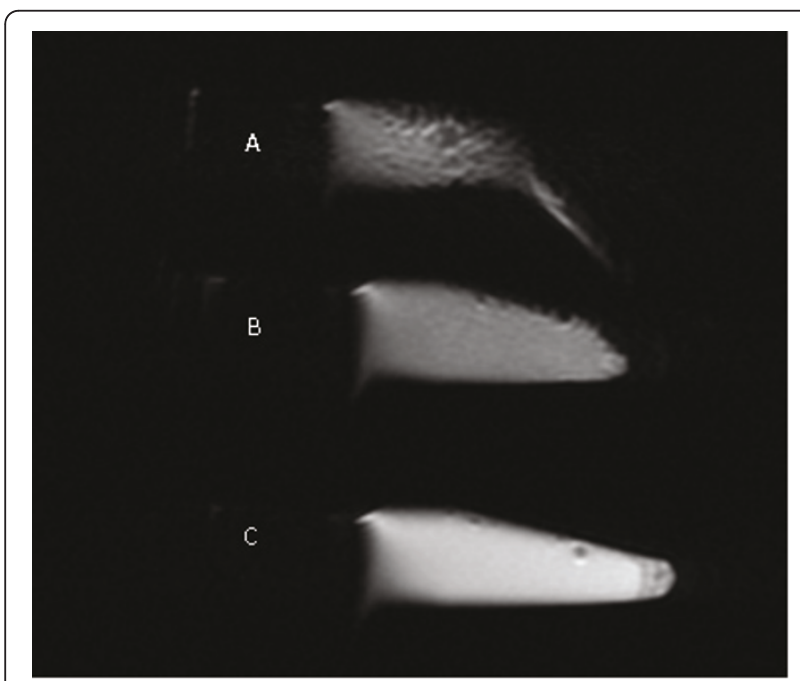

Figure 5 MR imaging of MGC803 cells and HDF cells. A MGC803 cells with anti-BRCAA1-FMNPs. B:HDF cells with antiBRCAA1-FMNPs. C: MGC803 cells with only FMNPs.
FMNPs nanoprobes were preferentially accumulated in the tumor tissues. Indeed based on the results in Figure $6 \mathrm{~B}$, the higher tumor to background ratio (TBR) value highly suggested that as-prepared nanoprobes preferentially accumulated in tumor tissues compared to normal control tissues. This was confirmed in fluorescence images, which showed that the fluorescence signal of asprepared nanoprobes in the tumor site was strongest among all mice organs as shown in Figure 6C. In addition, after $12 \mathrm{~h}$ post-injection of anti-BRCAA1-FMNPs nanoprobes, fluorescence intensity in tumor was still observed clearly, while the uptake of prepared nanoprobes in normal organs was not obvious. These data highly suggest that prepared nanoprobes can target highly efficiently tumor tissues inside nude mice loaded with gastric cancer. We also observed that those nanoprobes in the whole mouse body almost completely disappeared at $12 \mathrm{~h}$ post-injection, we also detected the nanoprobes exited out from the cholecyst system (data not shown), the time-dependent cholecyst clearance of nanoprobes highly suggest that as-prepared nanoprobes can not stay inside nude mice for longer time, thus, asprepared nanoprobes own good bio-safety.

\section{Pathological analysis of tumor and important organs}

In vitro evaluation of excised major tissues including liver, lung, spleen, kidney, and heart, as well as the tumor, indicated that the anti-BRCAA1-FMNPs probes were mainly up-taken by the tumor tissues, which exhibited strong fluorescence signals, as shown in Figure 7, whereas other tissues including liver, lung, spleen and heart up-took antiBRCAA1-FMNPs nanoprobes very less, which furtherly indicates that as-prepared BRCAA1-FMNPs nanoprobes can target gastric cancer tissues. We also used HE staining to check all organs, no obvious damages were observed in important organs [see additional file 1].

\section{As-prepared nanoprobes for MR Imaging of nude mice loaded with gastric cancer}

In vivo MR imaging was performed on nude mice loaded with subcutaneous gastric cancer at $12 \mathrm{~h}$ postinjection. Representative images of T2 maps were shown in Figure 8, after injecting the nanoprobes, a significant change in signal intensity was observed in some regions of tumors, indicating that there existed accumulation of the nanoprobes in tumor site as shown in Figure 8B, as the arrow showed. As a control, after the mice model with gastric cancer were injected FMNPs for $12 \mathrm{~h}$, the mice were performed MR imaging, which did not show intensive signal in tumor area (Figure 8A).

\section{Potential mechanism of targeting imaging}

In recent years, molecular imaging technologies have been used for real-time and non-invasive imaging of in 

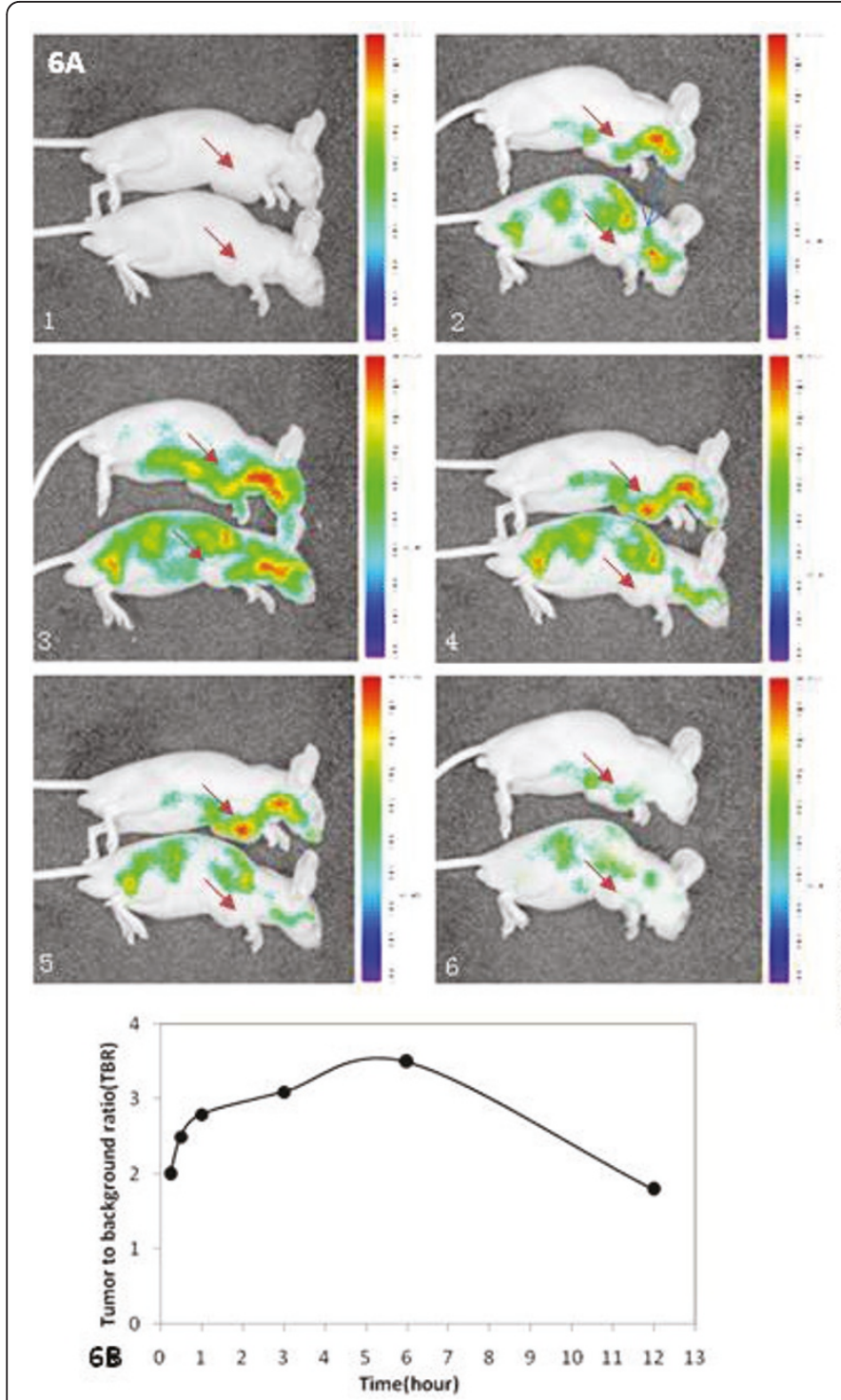

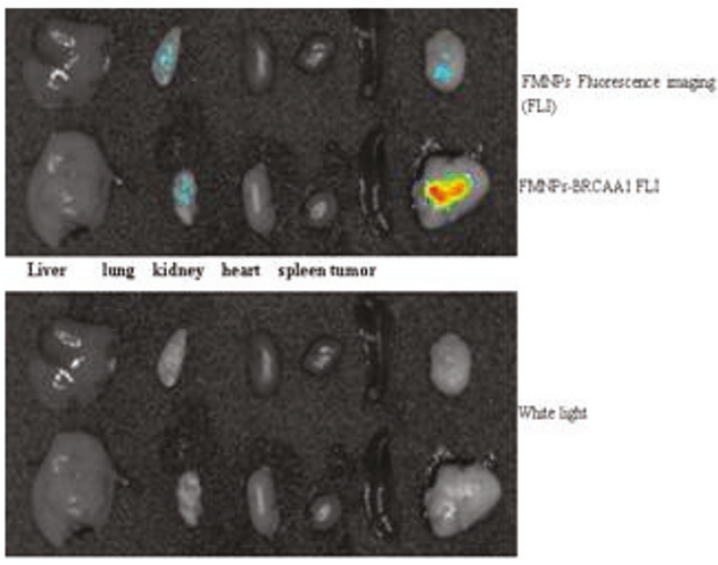

$6 \mathrm{C}$

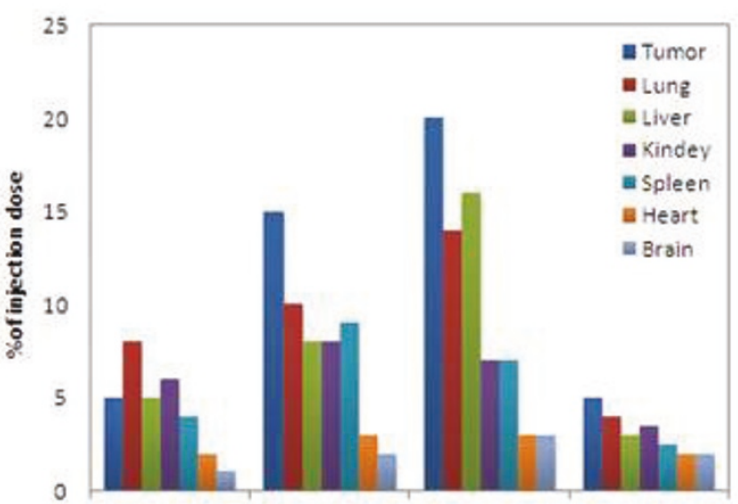

6 Time Post Injection anti-BRCAA1-FMNPs (hours) ${ }^{12}$

Figure 6 In vivo fluorescence images of tumor accumulation and tissue distribution for FMNPs-BRCAA1 nanoparticles in MGC803 human gastric tumor-bearing athymic nude mice. A, In vivo fluorescence images of athymic nude mice bearing. MGC803 human gastric tumor was obtained after injection of FMNPs-BRCAA1 nanoparticles at different time point. The tumor location is specified with an arrow. A-1: 0 h, A-2:0.5 h, A-3:1 h, A-4:3 h, A-5:6 h, A-6:12 h. B, TBR [Tissue to background (muscle) ratio] value. The TBR value was determined as follows: TBR $=($ Tumor signal-background signal)/(background signal). C, Ex vivo fluorescence images of dissected organs and tumor of mice bearing MGC803 human gastric tumor sacrificed at $12 \mathrm{~h}$ after injection of FMNPs-BRCAA1 nanoparticles. The fluorescence images of dissected organs and tumor were obtained using a fluorescence imaging technique with a $630 \mathrm{~nm}$ emission filter. D, Biodistribution of anti- BRCAA1-FMNPs in mice after intravenous injection. Several time points after injection, iron amounts in tissue samples were evaluated by ICP mass spectrometry $(n=3)$.

vivo tumor tissues [39-43]. For example, quantum dots, due to their unique photoluminescent properties, have been used for bio-labeling and fluorescent imaging [11-13,33,43], but quantum dots' toxicity limited their application in human body, so far some safe quantum dots are being developed. Magnetic nanoparticles have also been used as contrast reagent for MR imaging $[15,33,36]$. At the same time, combination of two imaging modalities provides the advantages of both than using one method, which would provide comprehensive information on tumor localization, environment, and status.

In this study, we designed and prepared a novel imaging probe, which was composed of silicon-wrapped quantum dots and magnetic nanoparticles with the aim of enhancing their biocompatibility. Our results show that prepared silicon-wrapped quantum dots and magnetic nanoparticles are very stable, and own strong 


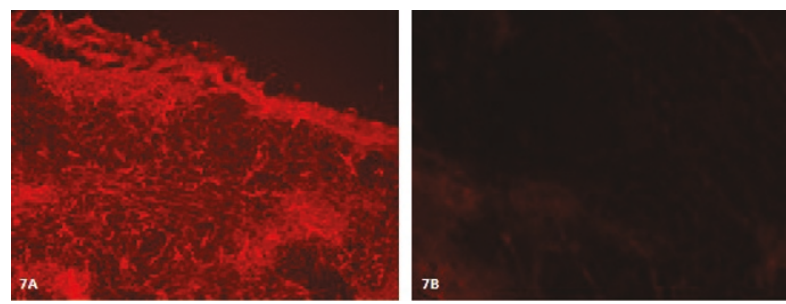

Figure 7 Result of Immunofluorescence Analysis. A, tumor tissue. B, liver. (Magnification $=\times 200$ ).

fluorescent signals and magnetic intensity. Using the strong fluorescent signals of as-prepared nanoprobes, we successfully obtained the fluorescent images of in vivo gastric cancer tissues with $5 \mathrm{~mm}$ in diameter in nude mice model. Using the strong magnetic signals of asprepared nanoprobes, we also successfully obtained MR images of in vivo gastric cancer tissues with $5 \mathrm{~mm}$ in diameter in nude mice model. Compared with previous reports, bigger size of tumor tissues $(>5 \mathrm{~mm})$ could be easily imaged by using fluorescent imaging and MRI imaging, as a contrast, our results showed that as-prepared nanoprobes can detect smaller size of tumor tissues (less $5 \mathrm{~mm}$ in diameter), which markedly improved the sensitivity of detection method. Our result also is the first time to report dual-modal targeting imaging of in vivo gastric cancer tissues.

How to target in vivo gastric cancer tissues is also a challengeable problem. Up to date, no specific gastric cancer biomarkers were reported. Although HER-2 protein was confirmed to have positive expression in 6-35\% of gastric cancer tissues [28-31], HER-2 protein also exhibits over-expression in many tumor tissues such as

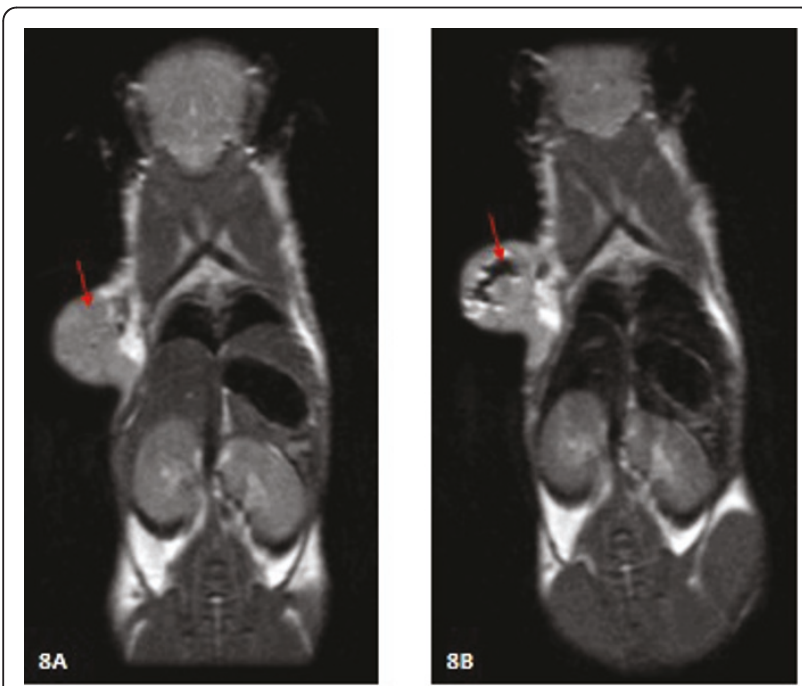

Figure 8 MRI image of mice. A, FMNPS without coupling BRCAA1 B, FMNPs coupled with BRCAA1 breast cancer, lung cancer, colon cancer, etc, therefore HER-2 should not be specific biomarker for gastric cancer. Our results showed that BRCAA1 antigen is only over-expressed in $64 \%$ or so of gastric cancer tissues from clinical surgery patients, we also confirmed that BRCAA1 antigen is over-expressed in some gastric cancer cell lines such as MKN-1, MKN-74, SGC-7901, KATO-III and MGC803 [6-9]. We used MGC803 cells to prepare nude mice model loaded with gastric cancer, and successfully observed that as-prepared nanoprobes preferentially accumulated in tumor tissues compared with normal control tissues, and as the post-injection time increased. We also observed that injected nanoprobes in the whole body exhibited the time-dependent clearance and the fluorescent signals gradually decreased as the time elapsed due to the liver-cholecyst excretion system and kidney clearness of as-prepared nanoprobes. Several reports showed that kidney only clear nanoparticles with $5 \mathrm{~nm}$ in diameter, in our study, we observed that as-prepared nanoprobes with $50 \mathrm{~nm}$ in diameter also could be cleared within $12 \mathrm{~h}$. This concrete mechanism is under way.

Nanoprobe biosafety is also an important problem [44], which decides the application prospect of as-prepared nanoprobes. Our results fully showed that as-prepared nanoprobes did not damage important organs including liver, kidney, heart, lung, etc, also did not exhibit long-term staying in important organs, which highly suggest that as-prepared nanoprobes own good biocompatibility, and have great potential in applications such as dual model imaging and selective therapy of early gastric cancer.

\section{Conclusion}

We successfully prepared a novel anti-BRCAA1-FMNPs nanoprobes, which can be used for in vivo two modal imaging such as fluorescent imaging and magnetic resonance imaging, and own an obviously specific targeting ability toward a gastric cancer tissues with $5 \mathrm{~mm}$ in diameter during $0.5 \mathrm{~h}$ and $12 \mathrm{~h}$ of post-injection, and own good biocompatibility. This should be first report. The as-prepared multifunctional nanoprobes also can be used for hyperthermia therapy of gastric cancer under in vitro alternating magnetic field irradiation, and have great potential in applications such as simultaneous imaging and targeting therapy of clinical gastric cancer in near future.

\section{Materials and methods}

\section{Preparation of anti-BRCAA1 monoclonal Antibodies}

Animal experiments were performed according to Guidelines for Animal Care and Use Committee, Shanghai Jiao Tong University. Monoclonal antibodies were prepared against a purified fusion protein BRCAA1. 
BALB/c female mice, 4-6 weeks old, were purchased from the Shanghai LAC Laboratory Animal Co. Ltd., Chinese Academy of Sciences (Shanghai, China). The mice were immunized by intraperitoneal injection with $50 \mu \mathrm{g}$ of purified BRCAA1 protein which was emulsified with an equal volume of Freund's complete adjuvant. Three further injections were administered using incomplete adjuvant every two weeks. Three days after the last injection, the spleen cells of the mice were harvested and fused with the Sp 2/0 mouse myeloma cell line. After 10-14 days, the culture supernatants were screened with an ELISA test in which the solid phase was coated with the recombinant BRCAA1 protein ( 2 $\mu \mathrm{g} / \mathrm{mL}$ ) used for the immunization. In the screening process, the monoclonal antibodies to bind with coated BRCAA1 protein were selected. By twice limiting dilution, positive colonies were subcloned. Ascitic fluids were harvested from the mice primed with a $0.5 \mathrm{~mL}$ intraperitoneal injection of Pristane and then injected with $10^{6}$ hybridoma cells. The class and subclass of each $\mathrm{mAb}$ were determined using a mouse monoclonal antibody isotyping kit (Hy Cult Biotechnology B.V., Netherlands). The mAbs were purified from the mouse ascetic fluids using a protein G-Sepharose 4FF (Pharmacia, Uppsala, Sweden) column according to the manufacturer's instructions to remove components which might interfere with the biopanning experiments. The antibody titers were determined by ELISA methods [45]. Finally one of prepared anti-BRCAA1 monoclonal antibodies was used as first antibody to stain 50 specimens of gastric cancer and control gastric mucous tissues, which were collected from Changzheng Hospital and No.1 People Hospital in Shanghai and identified by pathological examination.

\section{Preparation and Surface Functionalization of FMNPs}

Preparation of $\mathrm{Fe}_{3} \mathrm{O}_{4}$ nanoparticles was based on coprecipitation of ferrous and ferric ion solutions (1:2 molar ratio) [46-49]. CdTe nanocrystals were synthesized as follows according to our previous report: $\mathrm{CdCl}_{2}$ ( $5 \mathrm{mmol}$ ) was dissolved in $110 \mathrm{ml}$ of water, and 12 mmol of TGA were added under stirring, followed by adjusting the $\mathrm{pH}$ to 11 by dropwise addition of $1 \mathrm{M}$ $\mathrm{NaOH}$ solution. The mixed solution was placed in a three-necked flask deaerated by $\mathrm{N}_{2}$ bubbling for $30 \mathrm{~min}$. Under stirring, $2.5 \mathrm{mmol}$ of oxygen-free NaHTe solution was injected into the three-necked flask, which was freshly prepared from tellurium powder and $\mathrm{NaBH}_{4}$ (molar rate of $1: 2$ ) in water at $0^{\circ} \mathrm{C}$. The resulting solution was about $4 \mathrm{mg} / \mathrm{ml}$, and the $3.5 \mathrm{~nm}$ diameter product emitted with a maximum around $630 \mathrm{~nm}$. Fluorescent magnetic nanoparticles (FMNPs) were prepared using the reverse microemulsion approach. Before coupling the FMNPs with the BRCAA1, we first functionalized the surface functional group of FMNPs as carboxyl group. $95 \mathrm{~mL}$ ethanol and $2 \mathrm{~mL} 3$-Aminopropyltriethoxysilane (APS) were added to form a mixed solution and allowed to react at room temperature for $24 \mathrm{~h}$. The aminosilane-modified FMNPs were separated by permanent magnet and were washed with deionized water three times. Then redispersed the FMNPs- $\mathrm{NH}_{2}$ in $100 \mathrm{~mL}$ Dimethylformamide (DMF), added excessive succinic anhydride to form a mixed solution and react at room temperature for $24 \mathrm{~h}$. The carboxyl-modified FMNPs were separated by permanent magnet again and washed with deionized water three times.

\section{Preparation and characterization of BRCAA1 antibody- conjugated FMNPs}

We used two-step process to obtain stable antiBRCAA1-FMNPs conjugation [48,49]. 1.5 mg FMNPs$\mathrm{COOH}$ solution was dispersed in $2 \mathrm{~mL}$ pH7 PBS buffer, and was sonicated for $10 \mathrm{~min}$. Then we mixed $1 \mathrm{~mL}$ of fresh $400 \mathrm{mM}$ EDC and $100 \mathrm{mM}$ NHSS in pH 6.0 MES buffer and rotated it at room temperature for $15 \mathrm{~min}$. After this, the resulting solution was separated by magnetic field and $1 \mathrm{mg} / \mathrm{mL}$ BRCAA1 monoclonal antibody were added to the above mixture, stirred in dark place for $2 \mathrm{~h}$. To remove free BRCAA1, the residual reaction mixture was separated by magnetic field and the solid remaining was washed with $1 \mathrm{~mL}$ PBS buffer three times. Finally, $1 \mathrm{~mL} 0.05 \%$ Tween-20/PBS was added to the BRCAA1-FMNPs conjugation and the final bio-conjugation was stored at $4^{\circ} \mathrm{C}$. When we used, this BRCAA1-FMNPs conjugation should be diluted with PBS/0.05\% Tween-20. Then we used the Nano Drop device to quantify the coupling rate of BRCAA1 antibody with FMNPs-COOH. Before coupling reaction, we measured the total concentration of BRCAA1 antibody. After coupling reaction, we measured the BRCAA1 antibody concentration in residual reaction mixture and calculated the coupling rate according the equation:

Coupling $(\%)=(1$-Concentration of BRCAA1 antibody in residual reaction mixture/Total concentration of BRCAA1 antibody) $\times 100$.

The as-prepared nanoprobes and pure FMNPs were characterized by transmission electron microscopy and photoluminescence (PL) spectrometry, and Zeta potential analyzer.

\section{Nanoprobes for in Vitro targeting imaging of gastric cancer cells}

Gastric cancer cell line MGC803 cells with overexpressed BRCAA1 protein were used as target cells, human fibroblast cells without expressed BRCAA1 protein was used as control, were cultured and collected, and then were treated with $50 \mu \mathrm{g} / \mathrm{mL}$ BRCAA1-FMNPs nanoprobes and cultured in a humidified $5 \% \mathrm{CO}_{2}$ 
balanced air incubator at $37^{\circ} \mathrm{C}$ for $4 \mathrm{~h}$, meanwhile the MGC803 and human fibroblast cells were treated with FMNPs as the control group. Afterward, the cells were rinsed with PBS three times, and then fixed cells with $2.5 \%$ glutaraldehyde solution for $30 \mathrm{~min}$. For nuclear counterstaining, MGC803 were incubated with $1 \mathrm{mM}$ Hoechst 33258 in PBS for $5 \mathrm{~min}$. The cells were observed by fluorescence microscope (NIKON TS100F), and imaged by GE HDX 3.0T MR imaging instrument equipped with ParaVision 3.0 software.

We also used the Flow cytometer to evaluate the gastric cancer cell targeting ability of BRCAA1-FMNPs nanoprobes. MGC803 cells were treated with $50 \mu \mathrm{g} / \mathrm{mL}$ BRCAA1-FMNPs or FMNPs and harvested after $4 \mathrm{~h}$, and then we fixed the cells with $70 \%$ ethanol/PBS for 30 min on ice. Approximately $4 \times 10^{5}$ cells were centrifuged and resuspended with PBS, which were kept on ice until analysis. The number of cells which have been labeled with BRCAA1-FMNPs conjugation or FMNPs were analyzed by BD FACS Calibur Flow cytometer.

\section{Nanoprobes for fluorescence imaging of nude mode loaded with gastric cancer}

Animal experiments were performed according to Guidelines for Animal Care and Use Committee, Shanghai Jiao Tong University. Male athymic nude mice were obtained from Shanghai LAC Laboratory Animal Co. Ltd., Chinese Academy of Sciences (Shanghai, China). MGC-803 cells $\left(1 \times 10^{6}\right)$ were injected subcutaneously into the right anterior flank area of male nude mice with 4 to 5 weeks ages. Tumors were allowed to grow to a diameter of approximately $5 \mathrm{~mm}$. At that point, about $40 \mu \mathrm{g}$ BRCAA1-FMNPs nanoprobes was injected into the mice $(n=3)$ via the tail vein. Mice were respectively monitored in a non-invasive manner at $0.5,1,3,6$, and $12 \mathrm{~h}$ to get fluorescence images. Then, tumor and major organs were collected, and were placed on black papers, and subjected to IVIS Lumina imaging system (Xenogen) with emission wavelengths of $630 \mathrm{~nm}$. The fluorescence images [33] were acquired and total fluorescence flux for each sample was obtained. For the control experiment, mice $(n=3)$ were injected via tail vein with $40 \mu \mathrm{g}$ of FMNPs and subjected to optical imaging at various time points post-injection. Identical illumination settings (e.g., lamp voltage, filter, exposure time) were used in all animal imaging experiments.

\section{Nanoprobes for MR imaging of nude mice loaded with Gastric Cancer}

For MR imaging [33], gastric MGC-803 cells $\left(1 \times 10^{6}\right)$ were injected subcutaneously into the right anterior flank area of male nude mice $(n=3)$ with 4 to 5 weeks ages. After tumors reached approximately $5 \mathrm{~mm}$ in diameter, mice were injected with the BRCAA1-FMNPs nanoprobes. MR imaging was performed within $12 \mathrm{~h}$ after injections on animals anesthetized with $0.4 \%$ pentobarbital. MR imaging was performed using 3.0T field intensity by GE HDX 3.0T MR imaging instrument equipped with GE Signa Excite 3.0T MRI software. The imaging protocol consisted of coronal and transverse T2- weighted spin echo (SE) pulse sequences. To produce T2 maps, the following imaging parameters were used: $\mathrm{TR} / \mathrm{TE}=1000 / 10,20,30,40,50,60,70,80 \mathrm{~ms}$; $\mathrm{FOV}=8.0 \mathrm{~cm} ; \mathrm{NEX}=2$; slice thickness $=2.0 \mathrm{~mm}$. The mice $(n=3)$ model with gastric tumor performed MR imaging and injected FMNPs without labeling BRCAA1 were used for the negative control. Representative T2 maps of the animals loaded with tumors treated with FMNPs and BRCAA1-FMNPs, respectively. Coronal images showed a significant signal in BRCAA1-overexpressed tumors within $12 \mathrm{~h}$ after administration of the BRCAA1-FMNPs nanoprobes.

\section{Fluorescence microscopy observation and immunofluorescence analysis}

To compare the distributions of as-prepared nanoprobes in tissue and tumor, the mice in test group were euthanized after in vivo imaging. For histological evaluation, excised tumor and important organs were frozen and embedded by medium at $-20^{\circ} \mathrm{C}$, and then were sectioned into $8 \mu \mathrm{m}$ slices, which were used for fluorescence examination under inverted fluorescence microscope (Olympus IX71) equipped with digital camera and immunohistochemical study with BRCAA1 antibody. Digital images were processed with self-software (ImagePro Plus Version6.3). The important organ slices from heart, lung, kidney, brain and liver were analyzed by hematoxylin and eosin(HE) stain method.

\section{Statistical Analysis}

Each experiment was repeated three times in duplicate. The results were presented as mean \pm SD. Statistical differences were evaluated using the $t$-test and considered significance at $P<0.05$.

\section{Additional material}

Additional file 1: The results of important organs stained by HE A: heart; B:liver; C:spleen; D:lung; E:kidney; F: brain
Acknowledgements

This work was supported by the National Natural Science Foundation of China (No.20803040 and No.20471599), Chinese 973 Project (2010CB933901 and 2011CB933100), 863 Key Project (2007AA022004), New Century Excellent Talent of Ministry of Education of China (NCET-08-0350 and No.20070248050), Special Infection Diseases Key Project of China (2009ZX10004-311), Shanghai Science and Technology Fund (10XD1406100, $1052 \mathrm{~nm} 04100$ and No. 072112006-6) 


\section{Author details}

'Department of Bio-nano Science and Engineering, National Key Laboratory of Nano/Micro Fabrication Technology, Key Laboratory for Thin Film and Microfabrication of Ministry of Education, Institute of Micro-Nano Science and Technology, Shanghai Jiao Tong University, 800 Dongchuan Road, Shanghai 200240, China. ${ }^{2}$ Department of Surgery, Changzheng Hospital affiliated to Second Military Medical University, 151 Fengyang Road, Shanghai 20003, China.

\section{Authors' contributions}

KW and JR carried out the molecular genetic studies, KW and ZX participated in the sequence alignment and drafted the manuscript. CCB and HS carried out the immunoassays. DXC and JN participated in the design of the study and performed the statistical analysis. QRQ and $\mathrm{GHH}$ helped to collect clinical specimens from patients with gastric cancer, CLZ collected the normal control tissues, YFK prepared the FMNPs. All authors read and approved the final manuscript.

\section{Competing interests}

The authors declare that they have no competing interests.

Received: 7 February 2011 Accepted: 25 May 2011

Published: 25 May 2011

\section{References}

1. Bondy M: Cancer epidemiology and prevention. JAMA 2009, 301:1074-1075.

2. Jemal A, Siegel R, Ward E, et al: Cancer statistics. CA Cancer J Clin 2008 58:71-96.

3. $\mathrm{X} \cup \mathrm{A}, \mathrm{Li} \mathrm{S}$, Liu J, et al: Function of apoptosis and expression of the proteins $\mathrm{BCl}-2, \mathrm{p} 53$ and $\mathrm{C}-\mathrm{myc}$ in the development of gastric cancer. World J gastroenterol 2001, 7:403-406.

4. Cui $D$, Zhang $L$, Yan $X$, et al: A microarray-based gastric carcinoma prewarning system. World J Gastroenterol 2005, 11:1273-1282.4

5. Cui D, Yan X, Wang F, Su C: New strategy of cloning differentially expressed genes. Prog Biochem Biophys 2000, 27:362-364.

6. Cui D, Yan Wang F, Su C: Studies on differentially expressed genes of gastric cancer by mRNA differential display. Prog Biochem Biophys 2000, 27:379-382.

7. Cui D, Gao T, Jin G, Sun T, Sarai A: Cloning, chrosomal mapping and characterization analysis of BRCAA1. Ziran Zazhi 2003, 25:356-358.

8. Cui D, Jin G, Gao T, et al: Characterization of BRCAA1 and its novel antigen epitope identification. Cancer Epidemiol Biomarker \& Preven 2004, 13:1136-1142.

9. Huang $T$, Cui $D$, Lin $Q$, et al: Expression and its clinical significance of BRCAA1 protein in gastric cancer tissue and various differentiated cell lines. Chin J Gastroenterol 2007, 12:616-619.

10. Yang H, Cui D, Li Q, Huang T, Gao F, He R, Pan B, Shao J, You X, Liu FT: Effect of blocking BRCAA1 gene with siRNA on proliferation of MCF-7 cells and expression of $\mathrm{Rb}$ gene. Chinese Journal of Tumor Biotherapy 2006, 13:181-184.

11. Liu B, Cui D, Du T, LI Z, Song H, Yang H, Bao C, Gan H: Inhibitory effects of BRCAA1 gene silencing on gastric cancer MGC-803 cells and its possible mechanism. Chinese Journal of Tumor Biotherapy 2008, 15:522-526.

12. Medintz IL, Uyeda HT, Goldman ER, Mattoussi H: Quantum dot bioconjugates for imaging, labelling and sensing. Nature Mater 2005, 4:435-446.

13. Liu C: Research and development of nanopharmaceuticals in China. Nano Biomed Eng 2009, 1:1-12.

14. Cui $D$, Li Q, Huang $P$, et al: Real time PCR based on fluorescent quenching of mercaptoacetic acid-modified quantum dots for ultrasensitive specific detection of nucleic acids. Nano Biomed Eng 2010, 2:45-55.

15. Hu K, Jin B, Wang Q: QDs-cavity approach to controlled quantum teleportation of GHZ-like state. Nano Biomed Eng 2010, 2:109-116.

16. Cui D, Pan B, Zhang $H$, et al: Self-assembly of quantum dots and carbon nnaotubes for ultrasensitive DNA and antigen detection. Anal Chem 2008, 80:7996-8001.

17. Li Z, Huang $P$, Zhang $X$, et al: RGD-conjugated dendrimer-modified godl nanorods for in vivo tumor targeting and photothermal therapy. Molecular Pharmaceutics 2010, 7:94-104.
18. Ju S, Teng G, Zhang $Y$, et al: In vitro labeling and MRI of mesenchymal stem cells from human umbilical cord blood. Magn Reson Imaging 2006, 24:611-617.

19. Thompson J, Hayes L, Lloyd D: Modulation of firefly luciferase stability and impact on studies of gene regulation. Gene 1991, 103:171-177.

20. Hsu A, Hou L, Veeravagu A, et al: In vivo near-infrared fluorescence imaging of integrin ${ }_{\perp}^{\prime}$ v Molecular Imaging and Biology 2006, 8:315-323.

21. Sato A, Klaunberg B, Tolwani R: In vivo bioluminescence imaging. Comparative medicine 2004, 54:631-634.

22. Jain R, Munn L: Dissecting tumour pathophysiology using intravital microscopy. Nature Reviews Cancer 2002, 2:266-276.

23. Lyons S: Advances in imaging mouse tumour models in vivo. The Journal of Pathology 2005, 205:194-205.

24. Li L, Chen D, Zhang Y, et al: Magnetic and fluorescent multifunctional chitosan nanoparticles as a smart drug delivery system. Nanotechnology 2007, 18:405102

25. Yang $P$, Quan $Z$, Lu L, et al: Luminescence functionalization of mesoporous silica with different morphologies and applications as drug delivery systems. Biomaterials 2008, 29:692-702.

26. Huang $P$, Li Z, Yang D, et al: Photosensitizer-conjugated magnetic nanoparticles for in vivo simultaneous magnetofluorescent imaging and targeting therapy. Biomaterials 2011, 32:1-12.

27. Kong $Y$, Chen J, Gao F, et al: A Multifunctional Ribonuclease ${ }^{\odot} A^{\odot}$ IConjugated CdTe Quantum Dot Cluster Nanosystem for Synchronous Cancer Imaging and Therapy. Small 2010, 26:2367-2373.

28. Gravalos C, Jimeno A: HER2 in Gastric Cancer: A New Prognostic Factor and a Novel Therapeutic Target. Annals of Oncology 2008, 19:1523-1529.

29. Yan SY, Hu Y, Fan JG, et al: Clinicopathologic significance of HER-2/neu protein expression and gene amplification in gastric carcinoma. World J Gastroenterol 2011, 17:1501-1506.

30. Jørgensen JT: Targeted HER2 treatment in advanced gastric cancer. Oncology 2010, 78:26-33.

31. He R, You X, Shao J, et al: Core/shell fluorescent magnetic silica-coatedca Sinica nanoparticles for bioconjugation. Nanotechnology 2007, 18:315601.

32. You X, He R, Gao F, et al: Preparation and characterization of CdTe@SiO2 core/shell luminescent composite nanoparticles. Acta Chimica Sinica 2007, 65:561-565.

33. Cui $D$, Han $Y, L i$, et al: Fluorescent magnetic nanoprobes for in vivo targeted imaging and hyperthermia therapy of prostate cancer. Nano Biomed Eng 2009, 1:61-74.

34. Yang H, Li D, He R, et al: A novel quantum dots-based point of care test for syphilis. Nanscale Res Lett 2010, 5:875-881.

35. Ruan J, Shen J, Wang Z, et al: Efficient preparation and labeling of human induced pluripotent stem cells by nanotechnology. Int J Nanomed 2011, 6:1-11.

36. Yang $P$, Quan Z, Hou Z, et al: A magnetic, luminescent and mesoporous core-shell structured composite material as drug carrier. Biomaterials 2009, 30:4786-4795.

37. Insin N, Tracy J, Lee $H$, et al: Incorporation of Iron Oxide Nanoparticles and Quantum Dots into Silica Microspheres. Acs Nano 2008, 2:197-202.

38. Becker A, Hessenius C, Licha K, et al: Receptor-targeted optical imaging of tumors with near-infrared fluorescent ligands. Nature biotechnology 2001, 19:327-331.

39. Massoud T, Gambhir S: Molecular imaging in living subjects: seeing fundamental biological processes in a new light. Genes \& development 2003, 17:545.

40. Li L, Chen D, Zhang Y, et al: Magnetic and fluorescent multifunctional chitosan nanoparticles as a smart drug delivery system. Nanotechnology 2007, 18:405102.

41. Shi D, Cho H, Chen Y, et al: Fluorescent polystyrene-Fe3O4 composite nanospheres for in vivo imaging and hyperthermia. Advanced Materials 2009, 21:2170-2173.

42. Lyons S: Advances in imaging mouse tumour models in vivo. The Journal of Pathology 2005, 205:194-205.

43. Li Z, Hang P, Lin J, et al: Arginine-glycine-aspartic acid-conjugated dendrimer-modified quantum dots for targeting and imaging melanoma. J Nanosci Nanotech 2010, 10:4859-4867.

44. Cui D, Tian F, Ozkan CZ, Wang M, Gao H: Effects of single wall carbon nanotubes on human HEK293 cells. Toxicology Lett 2005, 155:77-83. 
45. Zhang B, Cheng J, Gong X, et al: Facile fabrication of multi-colors high fluorescent/superparamagnetic nanoparticles. Journal of colloid and interface science 2008, 322:485-490.

46. Chang $\mathrm{Q}, \mathrm{Zhu} \mathrm{L}, \mathrm{Yu} \mathrm{C}$, et al: Synthesis and properties of magnetic and luminescent Fe3O4/SiO2/Dye/SiO2 nanoparticles. Journal of Luminescence 2008, 128:1890-1895

47. Gerion D, Pinaud F, Williams S, et al: Synthesis and Properties of Biocompatible Water-Soluble Silica-Coated CdSe/ZnS Semiconductor Quantum Dots. J Phys Chem B 2001, 105:8861-8871.

48. Ruan J, Wang K, Song H, Xu X, Ji J, Cui D: Biocompatibility of hydrophilic silica-coated $\mathrm{CdTe}$ quantum dots and magnetic nanoparticles. Nanoscale Res Lett 2011, 6:299.

49. Wolcott A, Gerion D, Visconte M, et al: Silica-coated CdTe quantum dots functionalized with thiols for bioconjugation to IgG proteins. J Phys Chem B 2006, 110:5779-5789.

doi:10.1186/1477-3155-9-23

Cite this article as: Wang et al: BRCAA1 monoclonal antibody conjugated fluorescent magnetic nanoparticles for in vivo targeted magnetofluorescent imaging of gastric cancer. Journal of Nanobiotechnology 2011 9:23.

\section{Submit your next manuscript to BioMed Central} and take full advantage of:

- Convenient online submission

- Thorough peer review

- No space constraints or color figure charges

- Immediate publication on acceptance

- Inclusion in PubMed, CAS, Scopus and Google Scholar

- Research which is freely available for redistribution

Submit your manuscript at www.biomedcentral.com/submit 